\title{
Patient-Reported Outcomes-Guided Adaptive Radiation Therapy for Head and Neck Cancer
}

\author{
Sarah Weppler ${ }^{1,2 *}$, Harvey Quon ${ }^{3,4}$, Colleen Schinkel ${ }^{2,4}$, Adam Yarschenko ${ }^{2,5}$, \\ Lisa Barbera ${ }^{3,4}$, Nabhya Harjai ${ }^{6}$ and Wendy Smith ${ }^{1,2,4}$ \\ 1 Department of Physics and Astronomy, University of Calgary, Calgary, AB, Canada, ${ }^{2}$ Department of Medical Physics, Tom \\ Baker Cancer Centre, Calgary, AB, Canada, ${ }^{3}$ Department of Radiation Oncology, Tom Baker Cancer Centre, Calgary, \\ $A B$, Canada, ${ }^{4}$ Department of Oncology, University of Calgary, Calgary, AB, Canada, ${ }^{5}$ Department of Mechanical \\ Engineering, University of Calgary, Calgary, $A B$, Canada, ${ }^{6}$ Cumming School of Medicine, University of Calgary, \\ Calgary, $A B$, Canada
}

OPEN ACCESS

Edited by: Jason W. Sohn,

Allegheny Health Network,

United States

Reviewed by:

Jung Hun Oh

Memorial Sloan Kettering Cancer Center, United States

Francesco Ricchetti,

Sacro Cuore Don Calabria Hospital,

*Correspondence: Sarah Weppler Sarah.Weppler@albertahealthservices.ca

Specialty section: This article was submitted to

Radiation Oncology, a section of the journal

Frontiers in Oncology

Received: 16 August 2021 Accepted: 20 September 2021 Published: 19 October 2021

Citation:

Weppler S, Quon H, Schinkel C, Yarschenko A, Barbera L, Harjai N and

Smith W (2021) Patient-Reported Outcomes-Guided Adaptive Radiation Therapy for Head and Neck Cancer.

Front. Oncol. 11:759724. doi: 10.3389/fonc.2021.759724
Purpose: To identify which patient-reported outcomes (PROs) may be most improved through adaptive radiation therapy (ART) with the goal of reducing toxicity incidence among head and neck cancer patients.

Methods: One hundred fifty-five head and neck cancer patients receiving radical VMAT (chemo)radiotherapy (66-70 Gy in 30-35 fractions) completed the MD Anderson Symptom Inventory, MD Anderson Dysphagia Inventory (MDADI), and Xerostomia Questionnaire while attending routine follow-up clinics between June-October 2019. Hierarchical clustering characterized symptom endorsement. Conventional statistical approaches indicated associations between dose and commonly reported symptoms. These associations, and the potential benefit of interfractional dose corrections, were further explored via logistic regression.

Results: Radiotherapy-related symptoms were commonly reported (dry mouth, difficulty swallowing/chewing). Clustering identified three patient subgroups reporting: none/mild symptoms for most items (60.6\% of patients); moderate/severe symptoms affecting some aspects of general well-being (32.9\%); and moderate/severe symptom reporting for most items (6.5\%). Clusters of PRO items broadly consisted of acute toxicities, general well-being, and head and neck-specific symptoms (xerostomia, dysphagia). Dose-PRO relationships were strongest between delivered pharyngeal constrictor Dmean and patient-reported dysphagia, with MDADI composite scores (mean \pm SD) of $25.7 \pm 18.9$ for patients with Dmean $<50$ Gy vs. $32.4 \pm 17.1$ with Dmean $\geq 50$ Gy. Based on logistic regression models, during-treatment dose corrections back to planned values may confer $\geq 5 \%$ decrease in the absolute risk of self-reported physical dysphagia symptoms $\geq 1$ year post-treatment in $1.2 \%$ of patients, with a $\geq 5 \%$ decrease in relative risk in $23.3 \%$ of patients. 
Conclusions: Patient-reported dysphagia symptoms are strongly associated with delivered dose to the pharyngeal constrictor. Dysphagia-focused ART may provide the greatest toxicity benefit to head and neck cancer patients, and represent a potential new direction for ART, given that the existing ART literature has focused almost exclusively on xerostomia reduction.

Keywords: patient-reported outcomes, adaptive radiation therapy, head and neck cancer, dysphagia, xerostomia

\section{INTRODUCTION}

Standard-of-care (chemo)radiotherapy is associated with a high toxicity burden for many locally-advanced head and neck cancer patients. Physician assessments suggest that $\geq 30 \%$ of patients will experience grade 2 or worse radiationassociated dysphagia (1) with $\geq 35 \%$ experiencing grade 2 or worse xerostomia (2). Volumetric modulated arc therapy (VMAT) provides dose-sculpting capabilities to reduce incidental radiation doses to healthy tissues (2); however, decreases in tumor volume (3), weight loss (4), and other inter-fractional anatomical changes common among head and neck cancer patients may reduce treatment precision and increase toxicity $(5,6)$. Reduction of treatment-related side effects is increasingly important given the rise of HPV-related disease (7), as well as younger age and improved prognosis of these patients (8).

Adaptive radiation therapy (ART) adapts a patient's radiotherapy plan in response to inter-fractional anatomical changes to maintain target coverage and healthy tissue dose sparing objectives during the 6-7 week treatment course. ART may improve the therapeutic ratio of radiotherapy (3) and reduce treatment-related toxicities (5), but is resource intensive (9). Effective patient selection is therefore essential for ensuring that ART is feasible in a routine clinical setting. However, many open questions remain regarding patient selection: even in a broad sense, it is unclear which toxicity ART may most reduce.

When considering toxicity-reduction strategies, such as ART, patient-report outcomes (PRO) provide valuable insight into symptom burden. Physician assessments are essential for patient care but may underreport symptom severity relative to patient reporting (10). PROs help to fill the gap by providing the patient's perspective of the impact of symptoms and toxicity on daily patient life $(11,12)$. Examples of PRO instruments include the MD Anderson Symptom Inventory for Head and Neck Cancer (MDASI-HN) $(13,14)$, the MD Anderson Dysphagia Inventory (MDADI) (15) and the Xerostomia Questionnaire (XQ) (16). These instruments are widely used and score highly in reliability, validity, and responsiveness to changes over time (13-17).

In this study, we compare planned doses, delivered doses, and PROs (MDASI-HN, MDADI, and XQ) to identify which patientreported side-effects may be most improved by ART, and to estimate the associated toxicity benefit. It is our hope that these results will provide further structure to the development of ART workflows and effective patient-selection criteria.

\section{MATERIALS AND METHODS}

\subsection{Patient Inclusion Criteria}

Patients attending routine radiotherapy follow-up appointments between June and October 2019 were approached to complete a one-time paper-based PRO questionnaire in clinic. The questionnaire consisted of the MDASI-HN, MDADI, and XQ. Patients included in this study received treatment with radical VMAT (chemo)radiotherapy (66-70 Gy in 30-35 fractions). Patients were excluded if they were treated with a dose prescription less than $66 \mathrm{~Gy}$, did not receive CBCT imaging, or had a confirmed local-regional recurrence prior to survey completion. This study was approved by our institutional research ethics board (HREBA.CC-19-0119).

\subsection{Exposure Definition - Planned and Delivered Dose}

Planned organ-at-risk (OAR) dose parameter values were extracted from the patient's treatment plan. OAR planning objectives adhered to QUANTEC and other consensus recommendations and included: brainstem D0.03cc $\leq 54$ Gy (18); spinal cord D0.03cc $\leq 45$ Gy (19); ipsilateral and contralateral parotid gland Dmean $\leq 26$ Gy $(20,21)$; and pharyngeal constrictor Dmean $\leq 50$ Gy (22). Treatments were planned using the Eclipse Treatment Planning System, Versions 11 and 13 (Varian Medical Systems, Palo Alta, CA). Institutional image-guided radiation therapy utilized daily $\mathrm{kV}$-orthogonal imaging and weekly $\mathrm{kV}$-cone beam CT (CBCT) imaging (23).

Previously validated deformable image registration workflows allowed us to estimate delivered OAR doses (23). For each patient, we deformed a copy of the planning CT to reproduce the anatomical changes present in the last-acquired on-unit CBCT. We propagated contours through the corresponding deformation vector mapping, re-applied the patient's treatment plan, and recalculated dose in the treatment planning system. These doses served as a surrogate for total delivered dose. Assuming that patient anatomy was consistent with the final CBCT for all treatment fractions provided conservative estimates for the associations between dose and PROs. Quality assurance of this process assessed a representative set of cases (24), and ensured the propagated structures were geometrically (25) and dosimetrically (26) consistent with physician contours (23).

\subsection{Outcome Definition - Patient-Reported Outcome Instruments}

The MDASI-HN consists of 28 questions assessing core symptoms (13 items), head and neck-specific symptoms (9 items), and 
symptom interference on daily life (6 items) $(13,14)$. Each item is ranked from 0 to 10 with symptom burden interpreted as: none (item rating of 0 ); mild (1 to 4 ); moderate (5 to 6 ); or severe (7 to 10 ) (13). Summary symptom burden is defined by the maximum rating of any item within each subgroup: none (all items rated 0 ); mild (all items rated $<5$ with at least one item rated $\geq 1$ ); moderate (all items rated $<7$ with at least one item rate $\geq 5$ ); severe (at least one item rated $\geq 7)(27-29)$.

The MDADI contains 20 questions assessing physical swallowing ability (8 items), functional impact of swallowing dysfunction (5 items), emotional impact (6 items), and the general influence of swallowing ability on daily life (1 item) (15). Ratings for physical, functional, and emotional items are summed to produce the composite score (15). For this study, 5-point Likert-responses were normalized to 100 with higher scores indicating more severe symptoms. This provided greater comparability with the MDASI$\mathrm{HN}$ and XQ scoring systems. With this conversion, MDADI scores are interpreted as: minimal (summary score of 0 to 19), mild (20 to 39 ), moderate (40 to 59 ), severe (60 to 79 ), and profound (80 to 100 ) (30, 31). Differences in MDADI scores $\geq 10$ points are considered clinically relevant (32). References to MDADI moderate/severe scores below also include scores classified as "profound".

The XQ is an 8-item assessment of xerostomia symptoms while eating ( 4 items) and while not eating (4 items). Item scores are totaled and normalized to 100 (16). Symptom burden according to XQ responses was interpreted as: none/mild for scores $<50$ and moderate/severe for scores $\geq 50$ ).

\subsection{Covariates - Clinical Patient Characteristics}

Data for this study consisted of basic demographic and tumor factors abstracted from the patient's medical record. These included patient: age; gender; BMI; ECOG performance status; Charlson Comorbidity Index; tobacco/alcohol use; tumor site and stage; HPV status; and chemotherapy agent.

\subsection{Data Clustering, Statistical Analysis, and Logistic Regression Modelling} 2.5.1 Characterization of Patient-Reported Outcomes

Using Mann-Whitney U tests and Fisher's exact tests, we examined potential associations between clinical characteristics and PRO item and summary scores. Benjamini-Hochberg multiple testing corrections were applied with a false discovery rate of 5\% (33).

Hierarchical clustering tested for similarities in symptom reporting among PRO items and summary scores, as well as symptom burden among patients. This technique progressively groups items considered most similar, as represented in tree-like "dendrograms" (34). Similarities in PRO results were used to: characterize PRO reporting; verify dose-PRO associations among related PRO items; and identify similarities in patient symptoms to examine the effect of covariates.

\subsubsection{Associations Between Planned Dose, Delivered Dose and Patient-Reported Outcomes}

We stratified patients according to whether their OAR dose met $v s$. exceeded planning objective criteria. Differences in
PRO scores between these groups were compared using Mann-Whitney $U$ tests. Odds ratios indicated whether patients with OAR dose exceeding planning objectives had a greater likelihood of reporting moderate/severe symptoms, with significance from Fisher's exact tests. Tests were performed for both planned dose and delivered dose. For parotid gland doses, we compared the dose of the spared gland (i.e., the lesser of ipsilateral and contralateral gland Dmean values) with $\mathrm{PRO}$ results.

As moderate/severe symptoms persisting $\geq 1$ year after treatment are more likely to be permanent $(35,36)$, we further assessed differences in patients completing the PRO questionnaire $<1$ year $v s . \geq 1$ year post-treatment.

\subsubsection{Estimating the Benefit of Adaptive Replanning}

When delivered OAR doses were found to be strongly associated with PRO scores, we estimated the potential benefit of ART on patient-reported symptom severity. Systematic dose increases considered potentially correctable by replanning (dose "violations") were calculated relative to planning objectives and planned values, as relevant to clinical practice and QUANTEC guidelines. Additional tolerances accounted for random errors in estimated delivered doses to produce conservative estimates of ART benefit. For our given workflow, calculated increases in parotid gland dose exceeding $2.2 \mathrm{~Gy}$, and pharyngeal constrictor dose exceeding $0.75 \mathrm{~Gy}$ are likely to result from systematic changes in patient anatomy, as compared to daily setup uncertainties or deformable image registration error (23). For patients with planned doses meeting planning objectives,

Violation = delivered dose - planning objective - random error tolerance (1) For example, a patient with planned pharyngeal constrictor dose of $49.0 \mathrm{~Gy}$ and estimated delivered dose of 52.0 Gy would have a 1.25 Gy violation. For patients with planned doses exceeding planning objectives,

Violation $=$ delivered dose - planned dose - random error tolerance (2) Therefore, a patient with planned pharyngeal constrictor dose of 54.0 Gy and estimated delivered dose of 57.0 Gy would have a 2.25 Gy violation. Positive violation values indicate the amount of dose sparing achievable with adaptive dose corrections; patients with positive violations likely have increased risk of treatment-related side effects relative to that estimated at planning. Negative values indicate that: only minor dose increases occurred during treatment as a result of random effects; delivered structure dose corresponded to a relatively lowrisk of toxicity (i.e., delivered doses met the treatment planning objective); or that dose and corresponding toxicity risk decreased during treatment.

Logistic regression was used to model dose violations versus risk of moderate/severe symptom reporting. For each patient, the risk of moderate/severe symptom reporting was estimated for raw delivered doses and doses corrected back to planned values; corresponding differences in risk indicated the potential benefit, if any, of ART on patient-reported symptom severity.

All analyses were performed using $\mathrm{R}$ Version 3.6.0 (The $\mathrm{R}$ Foundation for Statistical Computing, Vienna, Austria). All statistical tests required $\mathrm{p} \leq 0.05$ for significance. 


\section{RESULTS}

\subsection{Cohort Characteristics and Characterization of Patient- Reported Outcomes}

225 patients completed the PRO questionnaires in clinic. After applying the inclusion/exclusion criteria, the final study cohort consisted of 155 patients. Table 1 provides cohort demographics and characteristics. MDASI-HN, MDADI, and XQ results are summarized in Figure 1. 60 patients completed the PRO questionnaire within their first year after treatment (median = 7 months, range $=2-11$ months), with the remaining 95 patients completing the questionnaire $\geq 1$ year post-treatment (28 months, 12-74 months).

Patients with lower initial BMI or poorer performance status more frequently reported moderate/severe fatigue, sadness, poorer activity, greater interference of symptoms with work, and poorer overall interference with daily life ( $\mathrm{p}<0.005$ for each) on the MDASI-HN. Greater T stage (T3-T4 disease) was significantly associated with higher MDADI composite summary scores $(p<0.005)$. No statistically significant differences occurred in clinical parameters for other MDASIHN, MDADI or XQ responses, including HPV status and time since treatment, according to Mann-Whitney $U$ tests and Fisher's exact tests.

Results of the hierarchical clustering are shown in Figure 2. PRO items were grouped according to: acute side-effects, general wellbeing, and xerostomia/dysphagia-related toxicities, with the latter combining various MDASI-HN, MDADI, and XQ items. The MDASI-HN dry mouth item strongly contributed to the MDASI-HN core and head and neck summary scores. Clustering indicated three general symptom profiles: none/mild symptoms for the majority of items (Cluster A, 60.6\% of patients); moderate/severe symptoms affecting some aspects of general wellbeing (Cluster B, 32.9\%); and moderate/severe symptom reporting for most items (Cluster C, 6.5\%). Patients in cluster $\mathrm{C}$ were younger on average ( 49.8 years, $\mathrm{p}=0.04)$, while patients in cluster A had a greater proportion of non-smokers $(46.8 \%, \mathrm{p}=$ 0.03). 6 of the 10 patients in cluster $C$, reporting moderate/severe symptoms for most items, had nasopharyngeal disease and greater planned and delivered brainstem dose although this was not found to be statistically significant. No other statistically significant differences persisted among the clinical, geometric, or dosimetric characteristics between clusters after multiple testing corrections.

\subsection{Associations Between Planned Dose, Delivered Dose and Patient- Reported Outcomes}

Table 2 summarizes the associations between OAR dose and PRO responses. Stratifying patients based on whether their planned pharyngeal constrictor doses met $v s$. exceeded the planning objective revealed statistically significant differences in MDADI composite, physical, and functional summary scores. These differences persisted for delivered pharyngeal constrictor dose, with additional statistical significance in
TABLE 1 | Cohort demographic and clinical characteristics.

\begin{tabular}{|c|c|}
\hline Parameter & $\begin{array}{l}\text { Full Cohort } \\
\qquad(\mathrm{n}=155)\end{array}$ \\
\hline Age in years, mean $( \pm S D)$ & $57.4(10.9)$ \\
\hline \multicolumn{2}{|l|}{ Gender, number (\%) } \\
\hline Male & $131(84.5 \%)$ \\
\hline Female & $24(15.5 \%)$ \\
\hline Initial BMI, mean $( \pm S D)$ & $28.1(5.6)$ \\
\hline ECOG, median (range) & $1(1-3)$ \\
\hline Charlson Comorbidity Index, median (range) & $4(2-8)$ \\
\hline \multicolumn{2}{|l|}{ Alcohol use, number (\%) } \\
\hline Never & $36(23.2 \%)$ \\
\hline Former & $12(7.7 \%)$ \\
\hline $\begin{array}{l}\text { Current - Light (males 0-15 drinks/week, females 0-10 } \\
\text { drinks/week) }\end{array}$ & $83(53.6 \%)$ \\
\hline $\begin{array}{l}\text { Current - Heavy (males >15 drinks/week, females >10 } \\
\text { drinks/week) }\end{array}$ & $24(15.5 \%)$ \\
\hline \multicolumn{2}{|l|}{ Tobacco use, number (\%) } \\
\hline Never & $63(40.7 \%)$ \\
\hline Cumulative - Light (0-20 pack-years) & $43(27.7 \%)$ \\
\hline Cumulative - Heavy (>20 pack-years) & 49 (31.6\%) \\
\hline \multicolumn{2}{|l|}{ Primary tumor location, number (\%) } \\
\hline Larynx & $7(4.5 \%)$ \\
\hline Hypopharynx & $3(1.9 \%)$ \\
\hline Oral Cavity & $3(1.9 \%)$ \\
\hline Oropharynx & $98(63.3 \%)$ \\
\hline Nasal Cavity & 7 (4.5\%) \\
\hline Nasopharynx & $26(16.8 \%)$ \\
\hline Unknown & $11(7.1 \%)$ \\
\hline \multicolumn{2}{|l|}{ T stage, number (\%) } \\
\hline $\mathrm{TO}-\mathrm{T} 2$ & $71(45.8 \%)$ \\
\hline $\mathrm{T} 3-\mathrm{T} 4$ & $73(47.1 \%)$ \\
\hline Tx & $11(7.1 \%)$ \\
\hline \multicolumn{2}{|l|}{ N stage, number (\%) } \\
\hline NO & $23(14.8 \%)$ \\
\hline N1 & $34(21.9 \%)$ \\
\hline $\mathrm{N} 2$ & $83(53.6 \%)$ \\
\hline N3 & $14(9.0 \%)$ \\
\hline NX & $1(0.7 \%)$ \\
\hline \multicolumn{2}{|l|}{ p16 status, number (\%) } \\
\hline Negative & $21(13.6 \%)$ \\
\hline Positive & $100(64.5 \%)$ \\
\hline Unknown & 34 (21.9\%) \\
\hline \multicolumn{2}{|l|}{ Chemotherapy agent, number (\%) } \\
\hline Carboplatin & $3(1.9 \%)$ \\
\hline Cetuximab & $13(8.4 \%)$ \\
\hline Cisplatin (Cisplatinum) & $128(82.6 \%)$ \\
\hline None & $11(7.1 \%)$ \\
\hline Time Since Treatment, median (range) & $\begin{array}{l}18 \text { months }(2-74 \\
\text { months) }\end{array}$ \\
\hline
\end{tabular}

emotional summary scores. Independently calculated odds ratios were statistically significant for MDADI physical and emotional scores with respect to both planned and delivered doses. Odds ratios associated with delivered doses exceeded those for planned doses, suggesting that delivered dose may be more strongly associated with these PRO summary scores. For MDADI composite scores, odds ratios had marginal significance for both planned dose $(\mathrm{OR}=2.02, \mathrm{p}=0.09)$ and delivered dose $(\mathrm{OR}=2.26, \mathrm{p}=0.06)$.

Furthermore, patients with doses meeting $v s$. exceeding the pharyngeal constrictor planning objective had significantly different MDADI scores across all summary items when reporting $\geq 1$ year after treatment completion, with respect to 


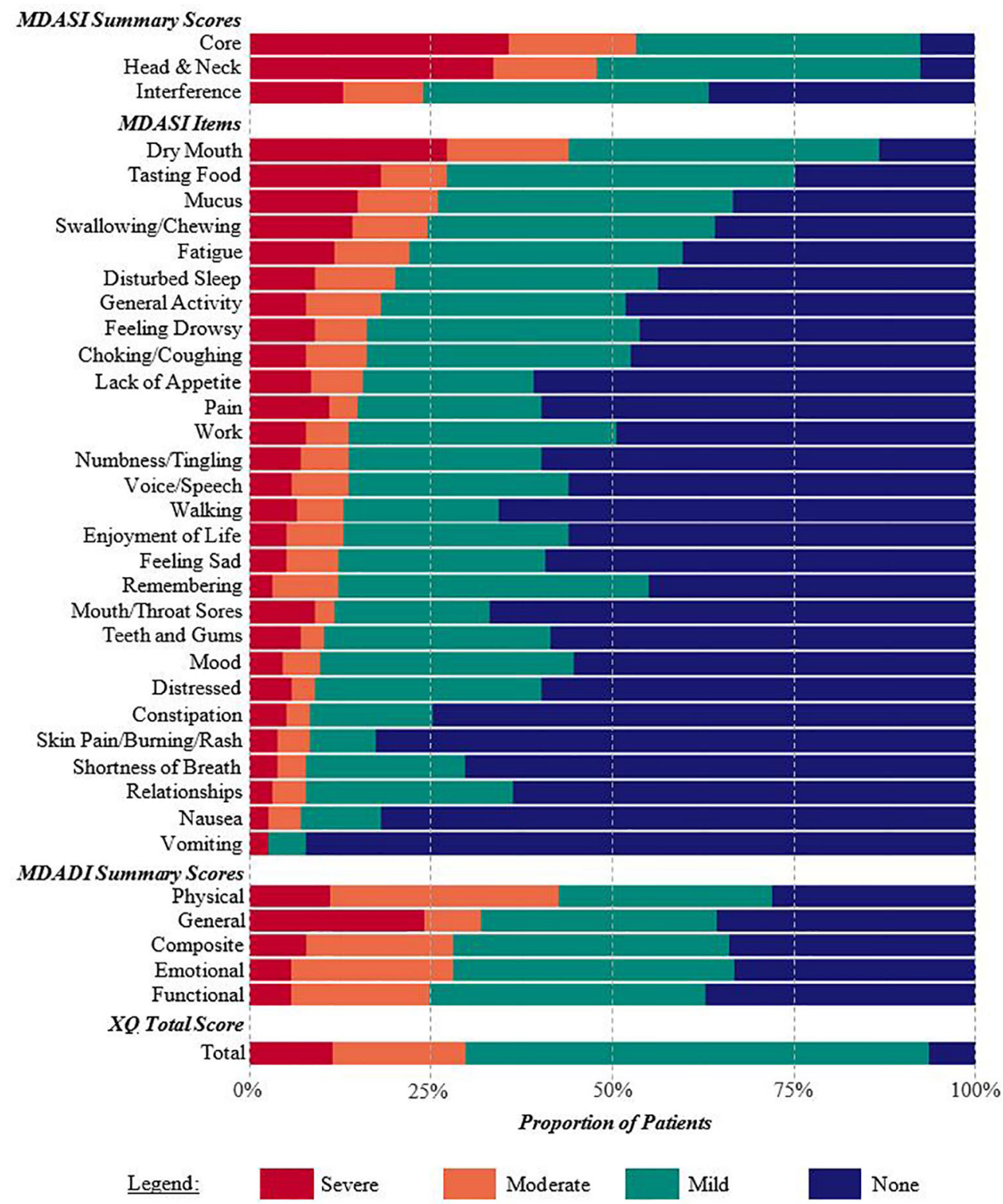

FIGURE 1 | Percentage of patients reporting none, mild, moderate, or severe symptoms on the MDASI-HN, MDADI, and XQ. Summary scores and individual items are listed according to the proportion of patients with moderate or severe symptoms. Xerostomia and dysphagia-related symptoms were commonly reported.

both planned and delivered doses; various MDADI summary scores had mean differences exceeding the 10 point threshold for clinical relevance (32). For planned doses, we observed differences in MDADI composite scores of 13.9; similarly, for delivered doses, we observed differences of 10.7. Mean differences exceeding 10 points also occurred for physical scores (16.3 with respect to planned doses; 13.3 for delivered dose) and general scores $(19.8 ; 14.8)$. This suggests that pharyngeal constrictor dose meaningfully stratifies patient symptom-reporting $\geq 1$ year post-treatment. Estimating odds ratios associated with $\mathrm{PRO}$ scores reported $\geq 1$ year post- treatment was limited by the small number of patients reporting moderate/severe symptoms with doses less than the planning objective.

Among patients with moderate/severe MDADI composite scores, $62.8 \%$ had planned pharyngeal constrictor doses exceeding the treatment planning objective, and $67.4 \%$ had delivered doses exceeding the objective (Figure 3A). In general, delivered doses exceeded planned doses for each patient (Supplementary Material). Although not statistically significant, Figure 3 indicates similar dose and PRO associations for MDASI-HN swallowing/chewing responses, 


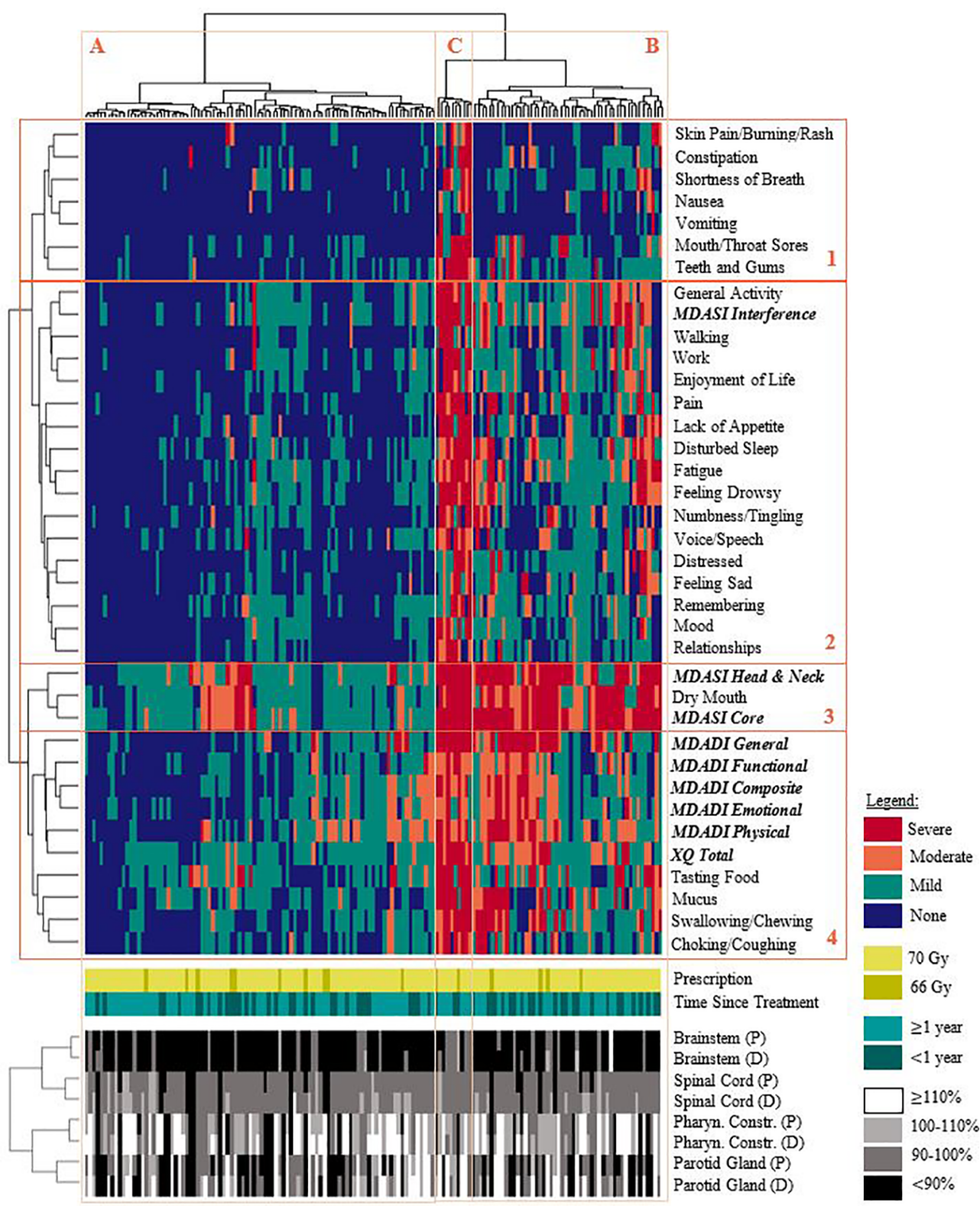

FIGURE 2 | Hierarchical clustering of patient-reported symptoms (none/mild/moderate/severe), prescription dose, time since completing treatment, and OAR dose. Each row (groups 1-4) represents a specific symptom or summary score and are clustered as: 1.) acute toxicities, 2.) general wellbeing, 3.) xerostomia-related summary scores, 4.) xerostomia and dysphagia-related symptoms. Each column represents a patient in the cohort; patients generally reported: (A) none/mild symptoms for most/all items, (B) moderate/severe symptom burden affecting some aspects of general wellbeing, (C) moderate/severe symptom reporting for most/ all items. Delivered dose generally exceeded planned dose. Note: healthy tissue doses are expressed as relative percentages of the planning objective. (P): planned dose. (D): delivered dose.

also observed for the MDASI-HN choking/coughing item (not shown), found to be related via cluster analysis. Associations appeared strongest among patients reporting $\geq 1$ year after treatment completion.

Patients with minimum parotid gland doses exceeding planning objectives had higher XQ scores, although this was not statistically significant (Table 2). No clear associations between parotid gland dose and patient-reported xerostomia symptoms were observed when considering patients in aggregate or according to $<1$ year $v s$. $\geq 1$ year posttreatment (Figure 3).

\subsection{Estimating the Benefit of Adaptive Replanning}

$55.6 \%$ of patients had non-negative pharyngeal constrictor dose violations. $33.1 \%$ of patients had pharyngeal constrictor dose 
TABLE 2 | Comparison of patient-reported symptom scores and dose, reported as mean (SD) for patients with dose meeting vs. exceeding planning objectives.

\begin{tabular}{|c|c|c|c|c|c|}
\hline \multirow[t]{2}{*}{ Toxicity/OAR (Obj.) } & \multirow[t]{2}{*}{ Relevant PROMs } & \multicolumn{2}{|c|}{ Planned Dose } & \multicolumn{2}{|c|}{ Delivered Dose } \\
\hline & & (<Obj./ $>$ Obj.) & OR $(95 \% \mathrm{Cl})$ & (<Obj./ $\geq$ Obj.) & OR $(95 \% \mathrm{Cl})$ \\
\hline Xerostomia/ & $\%$ Patients $(n=150)$ & $67.7 \%(100) / 32.3 \%(50)$ & $\mathrm{N} / \mathrm{A}$ & $55.5 \%(81) / 44.5 \%(69)$ & $\mathrm{N} / \mathrm{A}$ \\
\hline Parotid Glands & Average Dose & 20.1 Gy/30.8 Gy & $\mathrm{N} / \mathrm{A}$ & 19.3 Gy/31.9 Gy & $\mathrm{N} / \mathrm{A}$ \\
\hline \multirow[t]{9}{*}{ (Dmean $\leq 26$ Gy) } & MDASI Summary Scores and Relevant Items & & & & \\
\hline & Core & $5.1(3.2) / 4.9(3.2)$ & $0.73(0.38-1.39)$ & $5.3(3.3) / 4.8(3.0)$ & $0.93(0.49-1.77)$ \\
\hline & - Dry Mouth & $4.3(3.3) / 4.4(3.2)$ & $0.75(0.40-1.44)$ & $4.5(3.4) / 4.1(3.0)$ & $0.92(0.48-1.76)$ \\
\hline & Head \& Neck & $4.7(3.2) / 5.0(3.3)$ & $0.72(0.38-1.37)$ & $5.0(3.2) / 4.6(3.2)$ & $0.77(0.41-1.47)$ \\
\hline & - Swallowing/Chewing & $2.6(3.0) / 2.9(3.2)$ & $1.00(0.48-2.10)$ & $2.6(3.1) / 2.7(3.0)$ & $1.04(0.49-2.19)$ \\
\hline & - Taste & $3.1(3.1) / 3.3(2.8)$ & $0.88(0.43-1.81)$ & $3.2(3.2) / 3.0(2.7)$ & $0.89(0.43-1.83)$ \\
\hline & - Mucus & $2.7(2.9) / 2.7(3.5)$ & $1.00(0.48-2.08)$ & $2.9(3.0) / 2.5(3.2)$ & $1.03(0.49-2.14)$ \\
\hline & Interference & $2.7(2.7) / 2.4(3.0)$ & $0.65(0.30-1.37)$ & $2.7(2.9) / 2.4(2.8)$ & $0.78(0.37-1.64)$ \\
\hline & XQ Total Score & $32.3(23.4) / 37.2(26.9)$ & $1.66(0.80-3.46)$ & $32.8(24.0) / 35.1(25.4)$ & $1.70(0.81-3.57)$ \\
\hline Dysphagia/Pharyngeal & $\%$ Patients $(n=142)$ & $46.5 \%(59) / 53.5 \%(83)$ & $\mathrm{N} / \mathrm{A}$ & $42.6 \%(53) / 57.4 \%(89)$ & N/A \\
\hline \multirow[t]{14}{*}{ Constrictor (Dmean $\leq 50$ Gy) } & Average Dose & 44.3 Gy/56.5 Gy & $\mathrm{N} / \mathrm{A}$ & 44.1 Gy/57.1 Gy & $\mathrm{N} / \mathrm{A}$ \\
\hline & MDASI Summary Scores and Relevant Items & & & & \\
\hline & Core & $5.1(3.0) / 4.8(3.3)$ & $0.74(0.38-1.45)$ & $5.0(2.9) / 4.9(3.3)$ & $0.91(0.46-1.82)$ \\
\hline & Head \& Neck & $5.1(3.1) / 4.5(3.2)$ & $0.65(0.33-1.26)$ & $5.0(3.1) / 4.6(3.2)$ & $0.73(0.37-1.44)$ \\
\hline & - Swallowing/Chewing & $2.6(3.0) / 2.6(3.0)$ & $1.02(0.47-2.23)$ & $2.4(2.9) / 2.7(3.0)$ & $1.33(0.59-3.01)$ \\
\hline & - Choking/Coughing & $1.6(2.4) / 1.9(2.5)$ & $1.03(0.41-2.60)$ & $1.7(2.4) / 1.8(2.5)$ & $1.05(0.41-2.70)$ \\
\hline & - Taste & $2.9(3.1) / 3.1(3.0)$ & $0.97(0.46-2.06)$ & $2.8(3.1) / 3.2(3.0)$ & $1.03(0.48-2.22)$ \\
\hline & Interference & $2.7(2.8) / 2.4(2.8)$ & $0.59(0.27-1.29)$ & $2.8(3.0) / 2.3(2.7)$ & $0.55(0.25-1.20)$ \\
\hline & MDADI Summary Scores & & & & \\
\hline & Composite & 26.4 (18.7)/32.4 (17.2) & $2.02(0.90-4.50)$ & 25.7 (18.9)/32.4 (17.1) & $2.26(0.97-5.25)$ \\
\hline & Physical & $30.9(22.7) / 37.4(20.3)$ & $2.41(1.18-4.91)$ & $29.7(21.7) / 37.6(20.9)$ & $2.70(1.29-5.68)$ \\
\hline & Emotional & $25.2(18.2) / 30.6(18.7)$ & $2.52(1.11-5.72)$ & $24.6(18.5) / 30.6(18.4)$ & $2.87(1.20-6.87)$ \\
\hline & Functional & $20.6(19.4) / 26.7(18.7)$ & $1.70(0.73-3.92)$ & $20.6(21.4) / 26.3(17.5)$ & $1.63(0.69-3.85)$ \\
\hline & General & $25.9(30.0) / 33.4(30.8)$ & $1.41(0.68-2.93)$ & $26.4(31.1) / 32.6(30.2)$ & $1.25(0.60-2.64)$ \\
\hline
\end{tabular}

Bold entries indicate that mean values are statistically significant $(p \leq 0.05)$ according to Mann-Whitney tests, and that odds ratios are statistically significant ( $p \leq 0.05)$ according to Fisher's Exact tests. Obj.: treatment planning dose objective. OR: odds ratio denoting the odds of moderate/severe responses vs. none/mild responses for doses < Obj. vs. $\geq$ Obj.

violations exceeding $1 \mathrm{~Gy}$ (mean $=1.8 \mathrm{~Gy}$ in this cohort subgroup); $8.5 \%$ with increases exceeding 2 Gy (mean $=2.8$ Gy); and 3.5\% with increases exceeding 3 Gy (mean = 3.5 Gy).

Figure 4 shows the modelled risk of patients reporting moderate/severe MDADI physical scores (the most highly reported summary score) $\geq 1$ year post-treatment, with cohort results superimposed. For every $1 \mathrm{~Gy}$ increase in delivered dose, the absolute risk of moderate/severe symptom reporting increased by $1.5 \%$. Based on this model, we estimate that if doses were corrected back to planned values, absolute risk of selfreported dysphagia symptoms would decrease by $\geq 5 \%$ in $1.2 \%$ of patients. Given that the average absolute risk of self-reported dysphagia is $34.9 \%(\mathrm{SD}=9.3 \%)$, dose corrections may decrease relative risk by $\geq 5 \%$ in $23.3 \%$ of patients, $\geq 10 \%$ in $3.5 \%$ of patients, and $\geq 15 \%$ in $1.2 \%$ of patients. The model fit to MDADI composite scores is comparable, indicating a $1.6 \%$ decrease in absolute risk per Gy dose correction.

\section{DISCUSSION}

In this study, the strong relationship between delivered pharyngeal constrictor dose and patient-reported dysphagia is comparable to planned dose-PRO associations in the literature (37), yet further indicates that ART dose corrections may be beneficial for reducing dysphagia symptoms. In particular, our logistic regression models suggest that ART corrections may decrease the relative risk of patient-reported physical dysphagia symptoms by $\geq 5 \%$ in $23.3 \%$ of patients. We consider these estimates to be conservative. By using doses recalculated on the fraction of last $\mathrm{CBCT}$ acquisition to estimate total delivered dose, we make the assumption that patient anatomy was consistent with the last CBCT for all fractions; given that systematic changes in patient and tumor anatomy increase with progression through treatment, our calculations provide an upper bound on estimated inter-fractional dose increases. As corresponding increases in toxicity risk are the reciprocal of dose - calculated by dividing by estimated total delivered dose (e.g., probability of a side effect per Gy) - we obtain a conservative, lower estimate for ART-related toxicity reduction. Therefore, in practice, the toxicity-benefit of ART is likely to be greater than that indicated by our results. To demonstrate this, we performed an additional calculation under the assumption that accumulated delivered dose increases are half that estimated by using the last-acquired CBCT (e.g., assuming systematic anatomical changes increase linearly with time): we found that the absolute risk of moderate/severe MDADI physical scores increased by $1.6 \%$ per Gy ( $v s .1 .5 \%$ per Gy), with $2.3 \%$ ( $v$ s. $1.2 \%$ ) of patients having a $\geq 5 \%$ absolute decrease in the risk of selfreported dysphagia and $31.4 \%$ (vs. $23.3 \%$ ) of patients having a $\geq 5 \%$ relative decrease in risk.

Xerostomia-reduction is a primary focus of head and neck toxicity studies (2, 5, 38-41); however, dysphagia remains a significant toxicity concern affecting oral intake and healthrelated quality of life more adversely than xerostomia (42-44). 
A

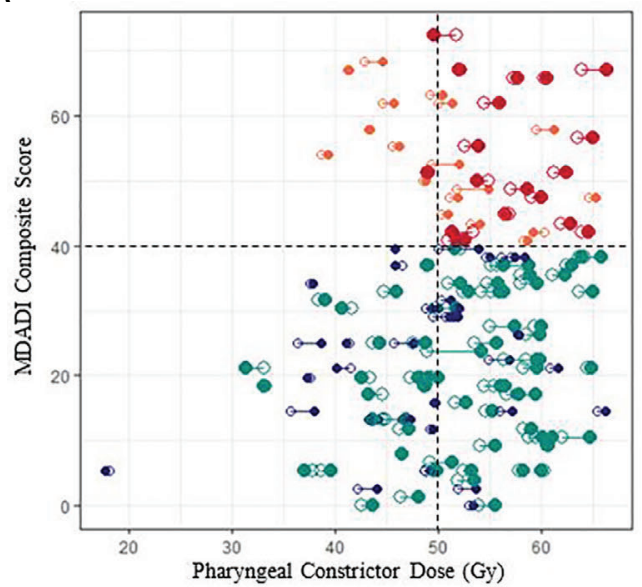

C

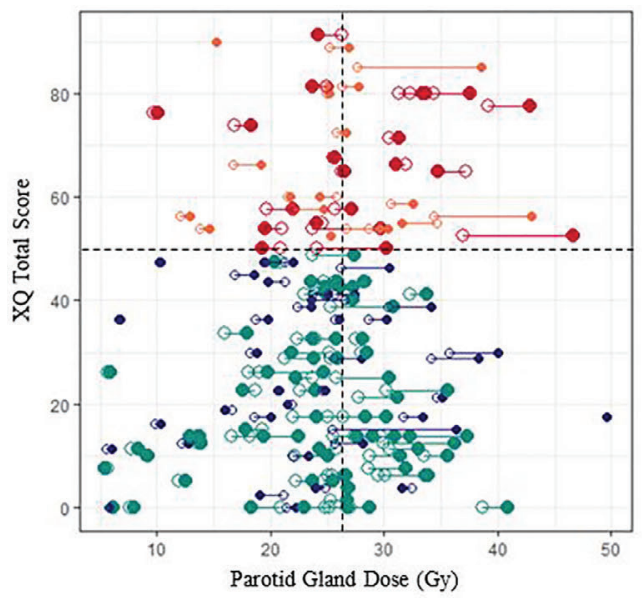

B

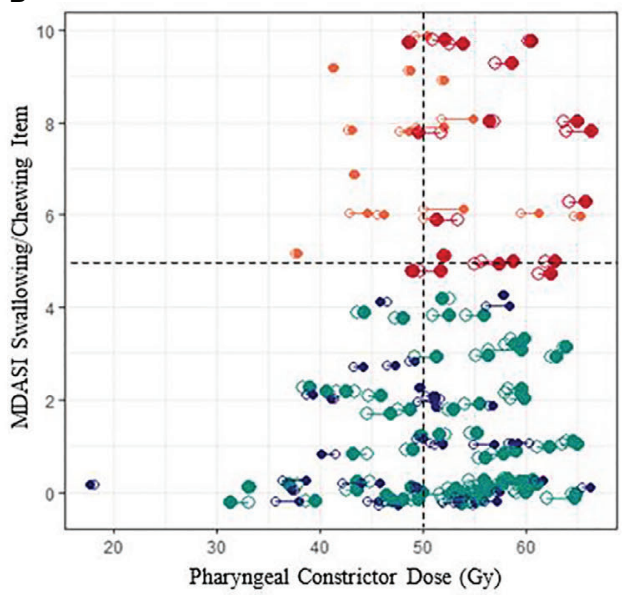

D

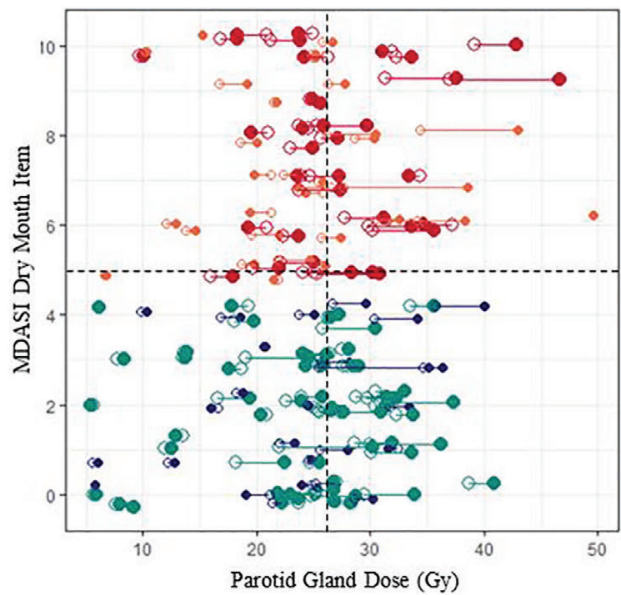

Legend: - None/Mild $<1$ year - Moderate/Severe $<1$ year $\bigcirc$ None/Mild $\geq 1$ year $\bullet$ Moderate/Severe $\geq 1$ year $\bigcirc$ Planned Dose Delivered Dose

FIGURE 3 | Examples of associations between paired planned and delivered OAR doses and PRO scores for each patient (joined by a horizontal line). (A, B) Pharyngeal constrictor doses of patients reporting moderate/severe dysphagia symptoms generally exceeded the planning objective of 50 Gy. (C, D) The relationship between parotid gland dose and patient-reported xerostomia symptoms was less clear. Random "jitter" up to \pm 0.3 has been added to MDASI-HN item scores to better visualize the data.

Dysphagia may result in nutritional deficiencies, weight loss, and feeding tube dependence as well as aspiration causing pneumonia and chronic bronchial inflammation (45). When safe to do so, higher prioritization of the pharyngeal constrictor may further reduce dysphagia symptoms (46). For cases where the pharyngeal constrictor is in close proximity to high dose volumes, as was common for our cohort, ART dose corrections may play an important role in dysphagia reduction.

To select patients for ART pharyngeal constrictor dose corrections, our previous work indicates the importance of pretreatment information, such as planned OAR doses and CTV volumes, and derives clinical guidelines from machine learning modeling (23). Pre-treatment patient selection may streamline ART workflows by allowing patients to be pre-booked for re-CTs and replanning, as compared to interfractional patient monitoring (e.g., assessing weight loss, decrease in face/neck diameter). While many dose-correction strategies exist in the field $(47,48)$, the work by Hamming-Vrieze et al. cautions against reducing GTV volumes (49), yet OAR doses may be reduced by correcting shifts in steep dose gradients resulting from anatomical changes.

PROs for our cohort are comparable with the existing literature $(28,29)$ and physician toxicity assessments $(1,2)$. Our violation formatting is consistent with QUANTEC and other consensus recommendations with respect to dose parameter types and planning objectives, however, future work may consider alternate dose parameter values and OAR such as submandibular and minor salivary glands. Submandibular glands were contoured for our cohort but were prone to deformable image registration errors in 


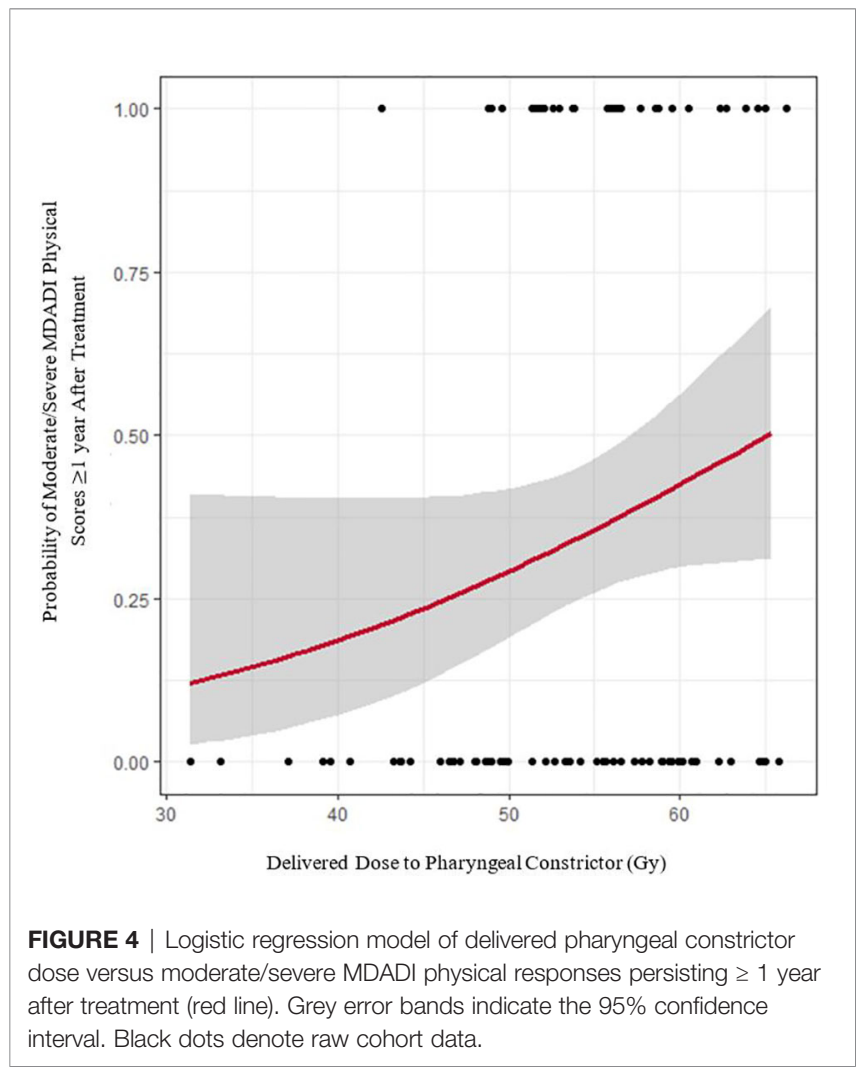

our dose estimation workflow, making delivered dose estimates unreliable in these structures (23). The literature indicates that while mean salivary gland dose is strongly associated with saliva flow rates and physician reporting, it is only weakly associated with XQ results $\geq 12$ months post-treatment (38) and may have contributed to the lack of dose-xerostomia associations for our cohort. Although not available for this cohort, OAR sub-contours may further refine dose-PRO associations and ART practices; the literature indicates that the superior pharyngeal constrictors are more strongly associated with late dysphagia (50), with the middle pharyngeal constrictors more strongly associated with acute dysphagia (50) and aspiration (51). Collecting PROs during the course of radiotherapy may build upon known associations between oral cavity dose, mucositis, and quality of life $(52,53)$.

Limitations of this study include a lack of baseline PRO measures and longitudinal data. We focus on doses to OAR that are most strongly associated with a given toxicity; however, salivary gland dose may further clarify dose-dysphagia associations (54). In estimating the potential benefit of correcting dose violations we make a conservative assumption that OARs may be corrected back to planned values (9). It is possible that corrective gains may be greater in this regard as well (9).

Future work on a larger study cohort may further investigate dose-PRO associations specific to head and neck tumor subsites (e.g., oropharyngeal $v s$. nasopharyngeal disease). We did not observe any statistically significant differences in PRO scores for this cohort with cancer subsite, which may be partially attributed to the similarity of prophylactic nodal volumes among patients of different subsites. As a result, we combined all head and neck cancer subsites into a single analysis; however, subtle differences among subsite groups may exist.

\section{DATA AVAILABILITY STATEMENT}

The datasets presented in this article are not readily available because of the conditions of ethics approval. Requests to access the datasets should be directed to Sarah.Weppler@albertahealthservices.ca.

\section{ETHICS STATEMENT}

The studies involving human participants were reviewed and approved by the Health Research Ethics Board of Alberta Cancer Committee. The patients/participants provided their written informed consent to participate in this study.

\section{AUTHOR CONTRIBUTIONS}

Overall study design was jointly proposed by SW, WS, CS, and HQ. SW and HQ coordinated data collection, including ethics approvals and survey circulation, assisted by AY and NH. SW performed data clustering, statistical analyses, and linear regression model development. LB provided guidance on the analysis of patient-reported outcomes data. SW prepared the initial manuscript with WS, CS, HQ, and LB. All authors contributed to the article and approved the submitted version.

\section{FUNDING}

This work was supported in part by the Natural Sciences and Engineering Research Council of Canada - Canada Graduate Scholarship (CGS-D) to SW, and the Calgary Foundation Cadmus Fund.

\section{ACKNOWLEDGMENTS}

The authors would like to thank Demetra Yannitsos, MPH, of the Tom Baker Cancer Centre for providing a foundational literature review for this study. Thank you also to Vicki Tran, of the University of Calgary, and Peter Chen, of the University of Alberta, for their assistance with survey collection.

An earlier version of this study was included in the first author's $\mathrm{PhD}$ thesis (55), completed at the University of Calgary, Canada.

\section{SUPPLEMENTARY MATERIAL}

The Supplementary Material for this article can be found online at: https://www.frontiersin.org/articles/10.3389/fonc.2021.759724/ full\#supplementary-material 


\section{REFERENCES}

1. Kamal M, Mohamed ASR, Volpe S, Zaveri J, Barrow MP, Gunn GB, et al. Radiotherapy Dose-Volume Parameters Predict Videofluoroscopy-Detected Dysphagia Per DIGEST After IMRT for Oropharyngeal Cancer: Results of a Prospective Registry. Radiother Oncol (2018) 128:442-51. doi: 10.1016/ j.radonc.2018.06.013

2. Nutting CM, Morden JP, Harrington KJ, Urbano TG, Bhide SA, Clark C, et al. Parotid-Sparing Intensity Modulated Versus Conventional Radiotherapy in Head and Neck Cancer (PARSPORT): A Phase 3 Multicentre Randomised Controlled Trial. Lancet Oncol (2011) 12:127-36. doi: 10.1016/S1470-2045 (10)70290-4

3. Barker JL, Garden AS, Ang KK, O'Daniel JC, Wang H, Court LE, et al. Quantification of Volumetric and Geometric Changes Occurring During Fractionated Radiotherapy for Head-and-Neck Cancer Using an Integrated CT/linear Accelerator System. Int J Radiat Oncol Biol Phys (2004) 59:960-70. doi: 10.1016/j.ijrobp.2003.12.024

4. Ahn PH, Chen CC, Ahn AI, Hong L, Scripes PG, Shen J, et al. Adaptive Planning in Intensity-Modulated Radiation Therapy for Head and Neck Cancers: Single-Institution Experience and Clinical Implications. Int $J$ Radiat Oncol Biol Phys (2011) 80:677-85. doi: 10.1016/j.ijrobp.2010.03.014

5. Brouwer CL, Steenbakkers RJHM, Langendijk JA, Sijtsema NM. Identifying Patients Who may Benefit From Adaptive Radiotherapy: Does the Literature on Anatomic and Dosimetric Changes in Head and Neck Organs at Risk During Radiotherapy Provide Information to Help? Radiother Oncol (2015) 115:285-94. doi: 10.1016/j.radonc.2015.05.018

6. Castelli J, Simon A, Lafond C, Perichon N, Rigaud B, Chajon E, et al. Adaptive Radiotherapy for Head and Neck Cancer. Acta Oncol (2018) 57:1284-92. doi: 10.1080/0284186X.2018.1505053

7. Chaturvedi AK, Engels EA, Pfeiffer RM, Hernandez BY, Xiao W, Kim E, et al. Human Papillomavirus and Rising Oropharyngeal Cancer Incidence in the United States. J Clin Oncol (2011) 29:4294-301. doi: 10.1200/JCO. 2011.36.4596

8. Ang KK, Harris J, Wheeler R, Weber R, Rosenthal DI, Nguyen-Tân PF, et al. Human Papillomavirus and Survival of Patients With Oropharyngeal Cancer. N Engl J Med (2010) 363:24-35. doi: 10.1056/NEJMoa0912217

9. Heukelom J, Fuller CD. Head and Neck Cancer Adaptive Radiation Therapy (ART): Conceptual Considerations for the Informed Clinician. Semin Radiat Oncol (2019) 29:258-73. doi: 10.1016/j.semradonc.2019.02.008

10. Falchook AD, Green R, Knowles ME, Amdur RJ, Mendenhall W, Hayes DN, et al. Comparison of Patient- and Practitioner-Reported Toxic Effects Associated With Chemoradiotherapy for Head and Neck Cancer. JAMA Otolaryngol Head Neck Surg (2016) 142:517-23. doi: 10.1001/jamaoto. 2016.0656

11. Jensen K, Bonde Jensen A, Grau C. The Relationship Between Observer-Based Toxicity Scoring and Patient Assessed Symptom Severity After Treatment for Head and Neck Cancer. A Correlative Cross Sectional Study of the DAHANCA Toxicity Scoring System and the EORTC Quality of Life Questionnaire. Radiother Oncol (2006) 78:298-305. doi: 10.1016/ j.radonc.2006.02.005

12. Hunter KU, Schipper M, Feng FY, Lyden T, Haxer M, Murdoch-Kinch CA, et al. Toxicities Affecting Quality of Life After Chemo-IMRT of Oropharyngeal Cancer: Prospective Study of Patient-Reported, ObserverRated, and Objective Outcomes. Int J Radiat Oncol Biol Phys (2013) 85:935-40. doi: 10.1016/j.ijrobp.2012.08.030

13. Cleeland CS, Mendoza TR, Wang XS, Chou C, Harle MT, Morrissey M, et al. Assessing Symptom Distress in Cancer Patients: The M. D. Anderson Symptom Inventory. Cancer (2000) 89:1634-46. doi: 10.1002/1097-0142 (20001001)89:7<1634::AID-CNCR29>3.0.CO;2-V

14. Rosenthal D, Mendoza T, Chambers M, Asper J, Gning I, Kies M, et al. Measuring Head and Neck Cancer Symptom Burden: The Development and Validation of the M.D. Anderson Symptom Inventory, Head and Neck Module. Head Neck (2007) 29:923-31. doi: 10.1002/hed.20602

15. Chen AY, Frankowshi R, Bishop-Leone J, Hebert T, Leyk S, Lewin J, et al. The Development and Validation of a Dysphagia-Specific Quality-of-Life Questionnaire for Patients With Head and Neck Cancer: The M. D. Anderson Dysphagia Inventory. Arch Otolaryngol Head Neck Surg (2001) 127:870-6.
16. Eisbruch A, Kim HM, Terrell JE, Marsh LH, Dawson LA, Ship JA. Xerostomia and Its Predictors Following Parotid-Sparing Irradiation of Head-and-Neck Cancer. Int J Radiat Oncol Biol Phys (2001) 50:695-704. doi: 10.1016/S03603016(01)01512-7

17. Ojo B, Genden EM, Teng MS, Milbury K, Misiukiewicz KJ, Badr H. A Systematic Review of Head and Neck Cancer Quality of Life Assessment Instruments. Oral Oncol (2012) 48:923-37. doi: 10.1016/j.oraloncology. 2012.03.025

18. Mayo C, Yorke E, Merchant TE. Radiation Associated Brainstem Injury. Int J Radiat Oncol Biol Phys (2010) 76:36-41. doi: 10.1016/j.ijrobp.2009.08.078

19. Kirkpatrick JP, van der Kogel AJ, Schultheiss TE. Radiation Dose-Volume Effects in the Spinal Cord. Int J Radiat Oncol Biol Phys (2010) 76:42-9. doi: 10.1016/j.ijrobp.2009.04.095

20. Deasy JO, Moiseenko V, Marks L, Chao KSC, Nam J, Eisbruch A. Radiotherapy Dose-Volume Effects on Salivary Gland Function. Int J Radiat Oncol Biol Phys (2010) 76:58-63. doi: 10.1016/j.ijrobp.2009.06.090

21. Moiseenko V, Wu J, Hovan A, Saleh Z, Apte A, Deasy JO, et al. Treatment Planning Constraints to Avoid Xerostomia in Head-and-Neck Radiotherapy: An Independent Test of QUANTEC Criteria Using a Prospectively Collected Dataset. Int J Radiat Oncol Biol Phys (2012) 82:1108-14. doi: 10.1016/ j.ijrobp.2011.04.020

22. Rancati T, Schwarz M, Allen AM, Feng F, Popovtzer A, Mittal B, et al. Radiation Dose-Volume Effects in the Larynx and Pharynx. Int J Radiat Oncol Biol Phys (2010) 76:64-9. doi: 10.1016/j.ijrobp.2009.03.079

23. Weppler S, Quon H, Schinkel C, Ddamba J, Harjai N, Vigal C, et al. Determining Clinical Patient Selection Guidelines for Head and Neck Adaptive Radiation Therapy Using Random Forest Modelling and a Novel Simplification Heuristic. Front Oncol (2021) 11:650335. doi: 10.3389/ fonc.2021.650335

24. Weppler S, Schinkel C, Kirkby C, Smith W. Data Clustering to Select Clinically-Relevant Test Cases for Algorithm Benchmarking and Characterization. Phys Med Biol (2020) 65(5):1-12. doi: 10.1088/1361-6560/ ab6e54

25. Brock KK, Mutic S, McNutt TR, Li H, Kessler ML. Use of Image Registration and Fusion Algorithms and Techniques in Radiotherapy: Report of the AAPM Radiation Therapy Committee Task Group No. 132: Report. Med Phys (2017) 44:e43-76. doi: 10.1002/mp.12256

26. Lim TY, Gillespie E, Murphy J, Moore KL. Clinically Oriented Contour Evaluation Using Dosimetric Indices Generated From Automated Knowledge-Based Planning. Int J Radiat Oncol Biol Phys (2019) 103:125160. doi: 10.1016/j.ijrobp.2018.11.048

27. Townes TG, Navuluri S, Pytynia KB, Gunn GB, Kamal MJ, Gilmore KR, et al. Assessing Patient-Reported Symptom Burden of Long-Term Head and Neck Cancer Survivors at Annual Surveillance in Survivorship Clinic. Head Neck (2020) 42:1919-27. doi: 10.1002/hed.26119

28. Eraj SA, Jomaa MK, Rock CD, Mohamed ASR, Smith BD, Smith JB, et al. Long-Term Patient Reported Outcomes Following Radiation Therapy for Oropharyngeal Cancer: Cross-Sectional Assessment of a Prospective Symptom Survey in Patients $\geq 65$ Years Old. Radiat Oncol (2017) 12:1-10. doi: 10.1186/s13014-017-0878-9

29. Gunn GB, Hansen CC, Garden AS, Fuller CD, Mohamed ASR, Morrison WH, et al. Favorable Patient Reported Outcomes Following IMRT for Early Carcinomas of the Tonsillar Fossa: Results From a Symptom Assessment Study. Radiother Oncol (2015) 117:132-8. doi: 10.1016/j.radonc.2015.09.007

30. Chen PH, Golub JS, Hapner ER, Johns MM. Prevalence of Perceived Dysphagia and Quality-of-Life Impairment in a Geriatric Population. Dysphagia (2009) 24:1-6. doi: 10.1007/s00455-008-9156-1

31. Ortigara GB, Schulz RE, Soldera EB, Bonzanini LIL, Danesi CC, Antoniazzi $\mathrm{RP}$, et al. Association Between Trismus and Dysphagia-Related Quality of Life in Survivors of Head and Neck Cancer in Brazil. Oral Surg Oral Med Oral Pathol Oral Radiol (2019) 128:235-42. doi: 10.1016/j.oooo.2019.05.009

32. Hutcheson K, Portwood M, Lisec A, Barringer D, Gries K, Lewin J. What Is a Clinically Relevant Difference in MDADI Scores Between Groups of Head and Neck Cancer Patients? Laryngoscope (2016) 126:1108-13. doi: 10.1002/ lary. 25778

33. Benjamini Y, Hochberg Y. Controlling the False Discovery Rate: A Practical and Powerful Approach to Multiple Testing. J R Stat Soc Ser B (1995) 57:289300. doi: 10.1111/j.2517-6161.1995.tb02031.x 
34. James G, Witten D, Hastie T, Tibshirani R. An Introduction to Statistical Learning With Applications in R. New York, NY: Springer (2013).

35. Memtsa P-T, Tolia M, Tzitzikas I, Bizakis J, Pistevou-Gombaki K, Charalambidou $\mathrm{M}$, et al. Assessment of Xerostomia and Its Impact on Quality of Life in Head and Neck Cancer Patients Undergoing Radiation Therapy. Mol Clin Oncol (2017) 6:789-93. doi: 10.3892/mco.2017.1200

36. Petkar I, Bhide S, Newbold K, Harrington K, Nutting C. Dysphagia-Optimised Intensity-Modulated Radiotherapy Techniques in Pharyngeal Cancers: Is Anyone Going to Swallow It? Clin Oncol (2017) 29:e110-8. doi: 10.1016/ j.clon.2017.02.002

37. Levendag PC, Teguh DN, Voet P, van der Est H, Noever I, de Kruijf WJM, et al. Dysphagia Disorders in Patients With Cancer of the Oropharynx Are Significantly Affected by the Radiation Therapy Dose to the Superior and Middle Constrictor Muscle: A Dose-Effect Relationship. Radiother Oncol (2007) 85:64-73. doi: 10.1016/j.radonc.2007.07.009

38. Miah AB, Gulliford SL, Clark CH, Bhide SA, Zaidi SH, Newbold KL, et al. Dose-Response Analysis of Parotid Gland Function: What Is the Best Measure of Xerostomia? Radiother Oncol (2013) 106:341-5. doi: 10.1016/ j.radonc.2013.03.009

39. Little M, Schipper M, Feng FY, Vineberg K, Cornwall C, Murdoch-Kinch CA, et al. Reducing Xerostomia After Chemo-IMRT for Head-and-Neck Cancer: Beyond Sparing the Parotid Glands. Int J Radiat Oncol Biol Phys (2012) 83:1007-14. doi: 10.1016/j.ijrobp.2011.09.004

40. Morgan H, Sher D. Adaptive Radiotherapy for Head and Neck Cancer. Cancers Head Neck (2020) 5:1-16. doi: 10.1186/s41199-019-0046-Z

41. Yang H, Hu W, Wang W, Chen P, Ding W, Luo W. Replanning During Intensity Modulated Radiation Therapy Improved Quality of Life in Patients With Nasopharyngeal Carcinoma. Int J Radiat Oncol Biol Phys (2013) 85:e4754. doi: 10.1016/j.ijrobp.2012.09.033

42. Kamal M, Barrow MP, Lewin JS, Estrella A, Gunn GB, Shi Q, et al. Modeling Symptom Drivers of Oral Intake in Long-Term Head and Neck Cancer Survivors. Support Care Cancer (2019) 27:1405-15. doi: 10.1007/s00520-0184434-4

43. Langendijk JA, Doornaert P, Verdonck-de Leeuw IM, Leemans CR, Aaronson NK, Slotman BJ. Impact of Late Treatment-Related Toxicity on Quality of Life Among Patients With Head and Neck Cancer Treated With Radiotherapy. J Clin Oncol (2008) 26:3770-6. doi: 10.1200/JCO.2007.14.6647

44. Ramaekers BLT, Joore MA, Grutters JPC, van den Ende P, de JJ, Houben R, et al. The Impact of Late Treatment-Toxicity on Generic Health-Related Quality of Life in Head and Neck Cancer Patients After Radiotherapy. Oral Oncol (2011) 47:768-74. doi: 10.1016/j.oraloncology.2011.05.012

45. Strojan P, Hutcheson KA, Eisbruch A, Beitler JJ, Langendijk JA, Lee AWM, et al. Treatment of Late Sequelae After Radiotherapy for Head and Neck Cancer. Cancer Treat Rev (2017) 59:79-92. doi: 10.1016/j.ctrv.2017.07.003

46. Nutting C, Rooney K, Foran B, Pettit L, Beasley M, Finneran L, et al. Results of a Randomized Phase III Study of Dysphagia-Optimized Intensity Modulated Radiotherapy (Do-IMRT) Versus Standard IMRT (S-IMRT) in Head and Neck Cancer. J Clin Oncol (2020) 38:6508-8. doi: 10.1200/JCO.2020. 38.15_suppl.6508

47. Bahig H, Yuan Y, Mohamed ASR, Brock KK, Ng SP, Wang J, et al. Magnetic Resonance-Based Response Assessment and Dose Adaptation in Human Papilloma Virus Positive Tumors of the Oropharynx Treated With Radiotherapy (MR-ADAPTOR): An R-IDEAL Stage 2a-2b/Bayesian Phase
II Trial. Clin Trans Radiat Oncol (2018) 13:19-23. doi: 10.1016/j.ctro. 2018.08.003

48. Heukelom J, Hamming O, Bartelink H, Hoebers F, Giralt J, Herlestam T, et al. Adaptive and Innovative Radiation Treatment FOR Improving Cancer Treatment outcomE (ARTFORCE); a Randomized Controlled Phase II Trial for Individualized Treatment of Head and Neck Cancer. BMC Cancer (2013) 13:1. doi: 10.1186/1471-2407-13-84

49. Hamming-Vrieze O, van Kranen SR, Heemsbergen WD, Lange CAH, van den Brekel MWM, Verheij M, et al. Analysis of GTV Reduction During Radiotherapy for Oropharyngeal Cancer: Implications for Adaptive Radiotherapy. Radiother Oncol (2017) 122:224-8. doi: 10.1016/j.radonc. 2016.10.012

50. Mazzola R, Ricchetti F, Fiorentino A, Fersino S, Giaj Levra N, Naccarato S, et al. Dose-Volumerelated Dysphagia After Constrictor Muscles Definition in Head and Neck Cancer Intensitymodulated Radiation Treatment. Br J Radiol (2014) 87:1-10. doi: 10.1259/bjr.20140543

51. Söderström K, Nilsson P, Laurell G, Zackrisson B, Jäghagen EL. Dysphagia Results From Multivariable Predictive Modelling on Aspiration From a Subset of the ARTSCAN Trial. Radiother Oncol (2017) 122:192-9. doi: 10.1016/ j.radonc.2016.09.001

52. Mazzola R, Ricchetti F, Fersino S, Fiorentino A, Giaj Levra N, di Paola G, et al. Predictors of Mucositis in Oropharyngeal and Oral Cavity Cancer in Patients Treated With Volumetric Modulated Radiation Treatment: A Dose-Volume Analysis. Head Neck (2016) 38:E815-9. doi: 10.1002/hed.24106

53. Franco P, Martini S, di Muzio J, Cavallin C, Arcadipane F, Rampino M, et al. Prospective Assessment of Oral Mucositis and Its Impact on Quality of Life and Patient-Reported Outcomes During Radiotherapy for Head and Neck Cancer. Med Oncol (2017) 34:1-8. doi: 10.1007/s12032-017-0950-1

54. van der Laan BFAM, van der Laan HP, Bijl HP, Steenbakkers RJHM, van der Schaaf A, Chouvalova O, et al. Acute Symptoms During the Course of Head and Neck Radiotherapy or Chemoradiation Are Strong Predictors of Late Dysphagia. Radiother Oncol (2015) 115:56-62. doi: 10.1016/j.radonc. 2015.01.019

55. Weppler S. J. Artificial Intelligence to Advance Adaptive Radiation Therapy (Unpublished doctoral thesis). University of Calgary, Calgary, AB (2020).

Conflict of Interest: The authors declare that the research was conducted in the absence of any commercial or financial relationships that could be construed as a potential conflict of interest.

Publisher's Note: All claims expressed in this article are solely those of the authors and do not necessarily represent those of their affiliated organizations, or those of the publisher, the editors and the reviewers. Any product that may be evaluated in this article, or claim that may be made by its manufacturer, is not guaranteed or endorsed by the publisher.

Copyright (C) 2021 Weppler, Quon, Schinkel, Yarschenko, Barbera, Harjai and Smith. This is an open-access article distributed under the terms of the Creative Commons Attribution License (CC BY). The use, distribution or reproduction in other forums is permitted, provided the original author(s) and the copyright owner(s) are credited and that the original publication in this journal is cited, in accordance with accepted academic practice. No use, distribution or reproduction is permitted which does not comply with these terms. 